The Chittagong Univ. J. Sci. 43(1): 75-92, 2021

\title{
Determinants and Distribution of Waiting Time for First Conception among Ever Married Women in Bangladesh
}

\author{
Md Abdul Karim* and Afia Azimoon \\ Department of Statistics, University of Chittagong, Chittagong-4331, Bangladesh \\ "Corresponding author: makstatcu@yahoo.com
}

Manuscript Submitted on 24/12/2020, Revised manuscript received on 31/08/2021, and accepted on 09/09/2021.

\begin{abstract}
Early age at first cohabitation has a major effect on childbearing because women, who marry early, have a longer period of exposure to the risk of becoming pregnant and higher fertility. In a traditional Bangladeshi society it is an urgent need to explore the timing of first conception because it plays a vital role in the determination of reproductive process and fertility. Therefore, the main purpose of this study is to explore meticulously the determinants and distribution of first conception wait among ever married women in Bangladesh, extracting data from Nationally Representative BDHS-2014 data set. In this study the overall first mean conception interval is found to be $19.13 \pm 20.80$ months with substantial variations by independent variables. The mean time required to conceive for the first time is found relatively low (15.54) among the respondents living in Chittagong division. The mean conception delay is found longer among those who have cohabited below age 15 years (24.36) and shorter (15.57) for the older marriage cohort. The results display that the distribution of first conception wait is positively skewed $\left(\beta_{1}=2.22\right)$ and leptokurtic $\left(\gamma_{2}=6.65\right)$. Life table analysis shows that $76 \%$ women become conceived within only three years of their first cohabitation. The tri-mean (T) and spread (S) of the first conception interval are found 16 and 24 months respectively. The findings of this study recommend that strict implementation of significant variables-legal age at first marriage; increase the literacy rate, improvement of socioeconomic status and reduction of overweight would be fruitful for normal timing of first conception and further reduction of fertility in Bangladesh.
\end{abstract}

Keywords: Cohabitation, conception, adolescent sterility, fertility, body mass index skewness, kurtosis, exposure interval, censored, tri-mean, life table.

DOI: https://doi.org/10.3329/cujs.v43i1.57337 


\begin{abstract}
অল্প বয়স, প্রথম কার্যকর প্রসবের ক্ষেত্রে বড় প্রভাব ফেলে কারণ মহিলারা, যারা খুব তাড়াতাড়ি বিবাহ করেন, তাদের দীর্ঘকালীন গর্ভবতী হওয়ার এবং জনউর্বরতার ঝুঁকি থাকে। ঐতিহ্যগত একটি বাংলাদেশী সমাজে প্রথম গর্ভধারনকালীন সময় অন্বেষণ করা জরুরী প্রয়োজন কারণ এটি প্রজনন প্রক্রিয়া এবং জনউর্বরতা নির্ধারণে গুরুত্বপূর্ন ভূমিকা পালন করে। সুতরাং, এই অধ্যয়নের মূল উদ্দেশ্য হনো জাতীয়ভাবে প্রতিনিধিত্বকারী BDHS-১৪ থেকে তথ্য আহরণ করে বাংলাদেশের কখনো না কখনো বিবাহিত মহিলাদের প্রথম গর্ভধারন সময়কাল নির্ধারক ও বিন্যাস নির্ভুলভাবে অনুসন্ধান করা। এ বিশ্লেষনে স্বতন্ত্র চলকের উল্লেখযোগ্য তারতম্যসহ প্রথম সামগ্রিক গর্ভধারনকালীন সময় পাওয়া যায় ১৯.১৩ড২০.৮০ মাস। চট্টগ্রাম বিভাগে বসবাসকারী উত্তরদাতাদের মধ্যে প্রথমবারের মতো গড় গর্ভধারণকালীন সময় পাওয়া যায় তুলনামূলকভাবে কম (১৫.৫৪ মাস)। একইভাবে, যাদের ১৫ বছরের নিচে কার্যকর বিয়ে হয়েছে তাদের গর্ভধারনকালীন সময় দীর্ঘতর (২৪.৩৬) এবং সাম্প্রতিক কোহর্ট এর জন্য তা অপেক্ষাকৃত সংক্ষিপ্ত (১৫.৫৭)। ফলাফলে দেখা যায় যে, প্রথম গর্ভধারনকালীন সময় এর বিন্যাস হলো ধনাত্মক বঙ্কিম ( $\beta$ s= ২.২২) এবং অতি সূচালু $\left(\gamma_{2}=৬ . ৬ ৫\right) ।$ লাইফ টেবিল বিশ্লেষণ দেখায় যে ৭৬\% মহিলা তাদের প্রথম কোহেবিটেশন এর ৩ বছরের মধ্যেই গর্ভধারন করে। প্রথম গর্ভধারনকালীন সময় এর ত্রি-গড় $(\mathrm{T})$ এবং স্প্রেড $(\mathrm{S})$ যথাক্রমে ১৬ এবং ২৪ মাস পাওয়া যায়। এই সমীক্ষার ফলাফলগুলি সুপারিশ করে যে, সারাদেশে তাৎপর্যপূর্ণ চলক- বিয়ের আইনী বয়সকে কঠোরভাবে প্রয়োগ করা, শিক্ষার হার বৃদ্ধিকরণ, আর্থ-সামাজিক অবস্থার উন্নিতকরণ এবং অতিরিক্ত ওজন হ্রাস করা হনে স্বাভাবিক প্রথম গর্ভধারনকালীন সময় এবং বাংলাদেশে জনউর্বরতা আরও হ্রাস করার জন্য ফলপ্রসূ হবে।
\end{abstract}

\title{
1. Introduction
}

The human reproduction starts from the onset of effective marriage and the timing of first conception. The first conception is an important and delightful event in the reproductive life of a woman. As such the analysis of the waiting time of first conception signifies couples reproductive characteristics and fertility at early stage of married life. The time, a woman takes to conceive for the first time after her first cohabitation is called the first conception wait. A conception delay is defined as the exposure months preceding, but not including, the month of conception, whereas conception wait or the time required to conceive includes that month as well [1]. That means the gap between marriage and first conception is termed as timing of first conception or first conception wait. It is very difficult to determine the exact age of attaining maturity and thus to estimate the actual waiting time for the first 
conception for such a female after enter into marital union [2]. A conception takes place during a given menstrual cycle only if (i) the cycle is ovulatory; (ii) insemination occurs during the fertile period in the middle of the cycle and (iii) fertilization results in recognizable conception [3]. Women's age at effective marriage and age at first conception are proximate determinants of fertility behavior. Usually, the later the age at effective marriage, the longer the interval between first cohabitation and first conception, the slower is the rate of population growth. A couple who intend to have one or two children may desire to lengthen the spacing between marriage and first conception in order to better establishment of their life and this would give more time for work experience before the child arrives. Thus, the lengthening of the interval between first cohabitation and first conception may be an important goal, but also may be in the interest of individual couple. Moreover, this interval has some unique characteristics to explore. Firstly, it is a basis for estimating fecundability. Secondly, usually couples would not like to use contraception to postpone first conception. Thirdly, it is free from the impact of post partum amenorrhea (PPA) and duration of breastfeeding. Fourthly, hardly there is any chance of memory lapse in reporting the time of first birth as it is the most pleasant event in the life of women [4]. Though, female age at first marriage in Bangladesh has been rising slowly since around the last decade of the preceding century, the current level is still quite low (15.86 years) in comparison to most of the low fertility countries. In some rural parts of Bangladesh, the first conception is usually delayed because of telephonic marriage and temporary separation of male partners due to occupation and staying overseas. Early child bearing contributes to population growth in two ways. First, in the absence of any intentional contraceptive practices, women who begin bearing children early in their life have more births than equally fecund women who begin so at older ages and secondly, child births occurring at younger ages imply a higher rate of fertility and population growth because of the shorter length of time between generations [5]. Furthermore, first conception is not only closely related to further childbearing but also closely associated with fertility. In populations with limited use of contraception, delays in 
age at first conception may have been one of the mechanisms to control fertility [6, 7]. Therefore, to understand the fertility pattern and reproductive behavior of Bangladeshi women, the in-depth study of timing of first conception along with its distribution is absolutely essential.

\section{Objectives of the Study}

The broad objective of this research work is to investigate the determinants and distribution patterns of timing of first conception among ever married women in Bangladesh because timing of first conception is one of the important determinants of reproductive span and fertility. Nonetheless, the explicit objectives of this study are as follows:

* To compute the overall mean along with confidence intervals and shape characteristics of timing of first conception ;

* To examine the mean timing of first conception by background characteristics of the respondents along with their variability and shape characteristics;

* To estimate the tempo and quantum of first conception interval along with other relevant measures by constructing an abridged life table including censored cases ;

* Finally, to recommend some suitable policies regarding timing and distribution of first conception along with age at first cohabitation and other determinants for safe reproductive health, safe motherhood, reduction of maternal and infant mortality, and in turn further reduction of fertility in the densely populated country of Bangladesh;

\section{Methodology}

This study is based on the data extracted from the 2014 Bangladesh Demographic Health Survey (BDHS). In this survey, out of 17863 respondents, 16,079 have been found in the birth cohorts and 1784 with parity zero. Among 16,079, respondents, 4021 were considered who gave at least one live birth in last three years preceding the survey to overcome the memory lapse of the respondents regarding exact date of 
birth of their first child, length of gestation and date of first cohabitation. Non-birth cohort is categorized into two groups; non birth with pregnancy and non-birth without pregnancy. In non-birth cohort 460 respondents was found pregnant at the time of interview. After inclusion of 460 with 4021 and excluding 801 owing to negative first conception intervals, thereafter 4280 respondents have been extracted for analyzing the first conception interval in Bangladesh. In addition, 1211 respondents were considered as censored as they have no birth at the date of the survey. Finally, considering the both birth and non-birth cohorts, total 5291 respondents has been extracted for multidimensional analysis.

The computational techniques of timing of first conception and exposure intervals of this study are given below:

\begin{tabular}{|l|l|}
\hline $\begin{array}{l}\text { First cohabitation to first } \\
\text { conception interval(closed first } \\
\text { conception interval) }\end{array}$ & $=\begin{array}{l}\text { Date of first Live } \\
\text { birth (Complete } \\
\text { Month Code) }\end{array}$
\end{tabular}$-\quad$\begin{tabular}{l}
9 \\
\hline
\end{tabular}$-$\begin{tabular}{l}
$\begin{array}{l}\text { Date of first } \\
\text { cohabitation } \\
\text { (Complete } \\
\text { Month Code) }\end{array}$ \\
\hline
\end{tabular}

\begin{tabular}{|l|}
\hline $\begin{array}{l}\text { Conception interval for non- } \\
\text { live birth }\end{array}=\begin{array}{l}\text { Date of first } \\
\text { pregnancy } \\
\text { terminated }\end{array}$ \\
\hline
\end{tabular}

\begin{tabular}{|l|}
\hline $\begin{array}{l}\text { length of } \\
\text { gestation }\end{array}$ \\
\hline
\end{tabular}$-\begin{aligned} & \text { Date of first } \\
& \text { cohabitation }\end{aligned}$

\begin{tabular}{|l|l|}
\hline $\begin{array}{l}\text { Conception interval for } \\
\text { currently pregnant women }\end{array}$ & $=$\begin{tabular}{|l|l|}
\hline Date of interview \\
\hline
\end{tabular}$-\begin{array}{l}\text { length of } \\
\text { gestation }\end{array}$
\end{tabular}$-\begin{aligned} & \text { Date of first } \\
& \text { Cohabitation }\end{aligned}$

\begin{tabular}{|l|l|}
\hline $\begin{array}{l}\text { Exposure Interval(open } \\
\text { interval) }\end{array}$ & $=$ Date of interview $-\begin{array}{l}\text { Date of first } \\
\text { cohabitation }\end{array}$ \\
\hline
\end{tabular}

After estimating the conception intervals from different cohorts, descriptive statistics of first conception waits have been calculated to compare the levels, variability and shape characteristics by available background characteristics of the ever married 
females. Since life table techniques an appropriate method to reduce the problems of truncated data, an abridge life table is constructed accordingly to estimate the tempo, quantum and also to examine the probability of having been first conception at different time points of exposure durations.

\section{Results and Discussion}

Timing of first conception plays a vital role in composition and structure of the population because it is one of the important segments of reproductive process which is also governed the level of fecundability and signals fertility at early stage of married life. Thus, the findings obtained regarding timing of first conception along with its differentials by background characteristics among ever married women in Bangladesh are presented in the Table 1 and probable interpretations are also given in the following sections accordingly because conception wait is highly influenced by socio-demographic, cultural and behavioral factors.

The findings in the Table 1 reveal that mean conception waits among Bangladeshi women is found to be 19.13 months with a marked variations by available background characteristics of the respondents. The shape characteristics first conception wait is observed to be positively skewed and leptokurtic. The overall mean conception wait is found to be longer in Bangladesh as compared to other low fertility populations. Bongaarts and Potter reported that the mean waiting time to conception ranging from 5 to 10 months. They also reported that the mean waiting time of first conception among German and Hutterite women were only 6 and 4.7 months respectively [8]. Jain reported that the mean conception wait among non contraceptive women of Taiwan was only 8.2 months [9]. In United States the mean time required to conceive was found to be 10.03 months. The median time of first conception was found only 6.4 months in Iran [10] and 18 months in an Indian study [11]. The same figure was estimated from Malaysian women [12]. Results in Table 1 demonstrate that illiteracy, poor socioeconomic condition, longer marital duration, early age at first cohabitation and overweight are the leading significant factors for the prolonged first conception interval among Bangladeshi women. 
It is evident from the Table 1 that place of residence of the sampled women is found one of the important factors in the variations of first cohabitation to first conception interval. Findings from the Table 1 demonstrate that mean conception interval for those who are living in urban areas is 19.87 months and it is 18.76 months for rural areas. The women living in urban areas have got greater chance for updating themselves, educating themselves which may be the cause of highest mean first conception interval probably due to the use of contraception before first conception and low age at first cohabitation among the females living in urban slum areas. On the other hand, women who are living in rural areas do not have the opportunities to have higher education due to some social and religious abstinence. Moreover, they have no intention to use contraception before first conception and also they got cohabitated at early age which leads them to be suffered from adolescent sterility, infant mortality and malnutrition, which lengthen their first conception interval.

Like place of residence marked variations in first conception intervals are also observed in different regions of residence of the females. The mean time required to conceive is found to be relatively shorter in Chittagong (15.54 months) followed by Sylhet (15.67) but fertility is higher in these two divisions (TFR: 2.5; 2.9). On the other hand the longer mean conception delay is found 22.60 months in Rajshahi followed by Barisal (20.36) but fertility is relatively lower in the above regions (TFR: $2.1 ; 2.2$ ). The higher mean age at first cohabitation and relatively lower use of contraception are the probable reasons for shorter conception interval in Sylhet and Chittagong divisions due to biological maturity. On the contrary, lower mean age at first cohabitation in Barisal (15.75 years) and Rajshahi (15.40 years) is one of the important reasons for longer mean conception interval due to adolescent sterility [13]. 
Table 1. Descriptive measures and Log-Rank test of first conception wait along with variability and shape characteristics by background characteristics of the respondents.

\begin{tabular}{|c|c|c|c|c|c|c|c|c|}
\hline \multirow[t]{2}{*}{$\begin{array}{c}\text { Background } \\
\text { Characteristics }\end{array}$} & \multirow[t]{2}{*}{ Mean } & \multirow[t]{2}{*}{$\begin{array}{l}\text { Standard } \\
\text { Deviation }\end{array}$} & \multirow[t]{2}{*}{$\beta_{1}$} & \multirow[t]{2}{*}{$\gamma_{2}$} & \multicolumn{2}{|c|}{$\begin{array}{c}95 \% \\
\text { Confidence } \\
\text { Interval }\end{array}$} & \multirow[t]{2}{*}{$\begin{array}{c}\text { Number of } \\
\text { Respondents }\end{array}$} & \multirow{2}{*}{$\begin{array}{c}\begin{array}{c}\text { Log-rank } \\
\text { (Mantel- } \\
\text { Cox) test }\end{array} \\
\chi^{2} \\
\end{array}$} \\
\hline & & & & & Max. & Min. & & \\
\hline \multicolumn{9}{|l|}{ Place of residence } \\
\hline Rural & 18.76 & 20.81 & 2.32 & 7.14 & 19.52 & 18.00 & 2861 & $21.25^{* * *}$ \\
\hline Urban & 19.87 & 20.79 & 2.03 & 5.75 & 20.95 & 18.79 & 1419 & \\
\hline \multicolumn{9}{|l|}{ Geographical region } \\
\hline Barisal & 20.36 & 19.60 & 2.12 & 6.24 & 22.05 & 18.66 & 514 & \multirow{7}{*}{$101.56^{* * *}$} \\
\hline Chittagong & 15.54 & 19.47 & 2.74 & 10.44 & 16.89 & 14.20 & 804 & \\
\hline Dhaka & 20.94 & 21.32 & 2.07 & 5.64 & 22.45 & 19.43 & 769 & \\
\hline Khulna & 20.29 & 20.69 & 2.08 & 5.52 & 22.06 & 18.52 & 525 & \\
\hline Rajshahi & 22.60 & 22.41 & 2.15 & 6.16 & 24.56 & 20.65 & 507 & \\
\hline Rangpur & 20.19 & 21.39 & 2.14 & 6.51 & 21.97 & 18.40 & 551 & \\
\hline Sylhet & 15.67 & 19.96 & 2.38 & 7.21 & 17.25 & 14.09 & 610 & \\
\hline \multicolumn{9}{|l|}{ Respondents' } \\
\hline No education & 27.46 & 28.84 & 1.73 & 3.02 & 30.09 & 24.83 & 463 & \\
\hline Primary & 18.38 & 19.41 & 2.15 & 6.38 & 19.50 & 17.25 & 1144 & \\
\hline Secondary \& above & 16.54 & 18.90 & 2.00 & 7.10 & 18.55 & 16.95 & 2673 & \\
\hline \multicolumn{8}{|l|}{ Marital duration } & $21.18^{* * *}$ \\
\hline $0-9$ & 15.71 & 15.92 & 1.75 & 3.70 & 16.28 & 15.14 & 3009 & \\
\hline $10-19$ & 25.62 & 26.33 & 1.94 & 4.33 & 27.18 & 24.06 & 1098 & \\
\hline $20+$ & 37.50 & 33.82 & 1.10 & 1.26 & 44.38 & 26.19 & 173 & \\
\hline \multicolumn{9}{|l|}{ Access to mass media } \\
\hline No access & 19.35 & 21.21 & 2.27 & 6.71 & 20.42 & 18.28 & 1502 & $17.04^{* * *}$ \\
\hline Have access & 19.00 & 20.58 & 2.20 & 6.61 & 19.77 & 18.24 & 2778 & \\
\hline
\end{tabular}




\begin{tabular}{|c|c|c|c|c|c|c|c|c|}
\hline \multirow[t]{2}{*}{$\begin{array}{c}\text { Background } \\
\text { Characteristics }\end{array}$} & \multirow[t]{2}{*}{ Mean } & \multirow[t]{2}{*}{$\begin{array}{l}\text { Standard } \\
\text { Deviation }\end{array}$} & \multirow[t]{2}{*}{$\beta_{1}$} & \multirow[t]{2}{*}{$\gamma_{2}$} & \multicolumn{2}{|c|}{$\begin{array}{c}95 \% \\
\text { Confidence } \\
\text { Interval }\end{array}$} & \multirow[t]{2}{*}{$\begin{array}{l}\text { Number of } \\
\text { Respondents }\end{array}$} & \multirow{2}{*}{$\begin{array}{c}\begin{array}{c}\text { Log-rank } \\
\text { (Mantel- } \\
\text { Cox) test }\end{array} \\
\chi^{2}\end{array}$} \\
\hline & & & & & Max. & Min. & & \\
\hline \multicolumn{9}{|l|}{$\begin{array}{l}\text { Socio economic } \\
\text { status }\end{array}$} \\
\hline Poor & 19.49 & 20.75 & 2.21 & 6.43 & 20.09 & 18.09 & 1650 & \multirow{3}{*}{$4.86^{*}$} \\
\hline Middle & 19.07 & 21.03 & 2.27 & 6.95 & 20.00 & 18.33 & 2437 & \\
\hline Rich & 18.33 & 18.31 & 1.45 & 2.58 & 21.52 & 16.35 & 193 & \\
\hline \multicolumn{9}{|l|}{$\begin{array}{c}\text { Age at first } \\
\text { cohabitation(Years) }\end{array}$} \\
\hline $10-14$ & 24.36 & 23.80 & 1.93 & 4.67 & 25.76 & 22.97 & 1116 & \multirow{3}{*}{$27.46^{* * *}$} \\
\hline $15-18$ & 17.78 & 19.81 & 2.37 & 7.84 & 18.57 & 17.00 & 2444 & \\
\hline $19+$ & 15.57 & 17.39 & 2.07 & 5.26 & 16.84 & 14.30 & 720 & \\
\hline \multicolumn{9}{|l|}{ Body mass index } \\
\hline Underweight & 17.69 & 19.14 & 2.17 & 6.00 & 18.89 & 16.50 & 986 & \multirow{3}{*}{$19.74^{* * *}$} \\
\hline Normal weight & 19.15 & 20.95 & 2.23 & 6.63 & 19.97 & 18.33 & 2514 & \\
\hline Overweight & 20.86 & 22.19 & 2.20 & 6.75 & 22.41 & 19.30 & 780 & \\
\hline Bangladesh & 19.13 & 20.80 & 2.22 & 6.65 & 19.75 & 18.50 & 4280 & \\
\hline
\end{tabular}

Note: $* * * \mathrm{p}<0.001 ; * \mathrm{p}<0.10$

Education has a significant interaction effect on timing of first conception and fertility. Table 1 presents a scenario of conception waits by educational levels of the respondents. Findings show that the conception wait is lower (16.54 months) among higher educated women than their illiterate counterparts (27.46 months). Illiterate women with low age at first marriage attributed to in-ovular cycles and adolescent sterility immediately after marriage which may delays their first conception due to biological immaturity. Moreover, it could be argued that highly educated women would perceive an early first conception due to late marriage.

Duration of marriage has found to be strong positive relationship with first conception interval. For instance, the mean length of first conception waits is found to be only 15.71 months for women with recent marriage cohort (up to 9 years) and it 
is about 37.5 months for those who have passed 20 years and above for their married life. The lower conception waits among the respondents with relatively shorter marital duration can be explained that they are newly married, highly fertile and the rate of coital frequency may also be higher. On the contrary, longer fist conception interval among the females having longer marital duration may be accompanied to prolonged separation of one of the partners, relatively less infertility, polygamy, and may also be a cause of obesity.

Respondents who have no access to mass media have higher mean first conception wait then who have no access. The respondents who have access to mass media have 19 months of first conception interval and who have no access, the corresponding figure is 19.35 months. Time required to conceive seems to be inversely related with socio economic status of the respondents. Women belong to high socioeconomic status take relatively shorter interval to conceive after marriage (18.33) as compared to those who belong to poor socioeconomic stratum (19.49). Adolescent infertility may be attributed to the women belong to poor economic status in the early stage of married life where age at first cohabitation is quite low.

Among the variables of interest, variation in ages of entry into marital union is one of the important factors for variations of first conception intervals, reproductive span and fertility trends across populations and individual populations [14]. In this study age at first cohabitation is found to have an inverse relationship with the marriage to first conception interval. The results in Table 1 indicate that conception wait tends to decrease with the increase of age at first cohabitation and the interval is prolonged (24.36) for whose age at first cohabitation is less than 14 years. The conception waits decreases consistently with the increase age at first cohabitation and is computed only 15.57 months for those whose mean age at marriage is 19 years and above. One of the important reasons for shorter mean conception waits for those who marry late is that they have already passed adolescent period and become adequately fertile to conceive. Moreover, couples with late marriage generally try to minimize the time gap for early family formation. As everyone considers that first conception is the transition marks to a woman into motherhood, so women have the desire to have their 
first conception as early as possible. Women who got married after the fulfillment of their legal age of marriage have lowest first conception interval, whereas who have childhood marriage have the highest first conception interval. Therefore, early age at first cohabitation implies that women are not ready to conceive on that age because of different complications like malnutrition and adolescent sterility.

The body mass index (BMI), which determined the nutritional status of the respondents, is also found to be positively correlated with first conception interval. Table 1 shows marked variations of first conception waits between malnourished and well nourished ever married females. Women belong to overweight category has longer mean conception interval of 20.86 months, whereas it is found 17.69 for those who belong to underweight stratum (17.69). Mean conception interval is found to be 19.15 months for those who have normal body mass index; the value is closest to the overall mean conception wait. This implies that health status is one of the important determinants influencing the first conception weight. Overweight during the time of conception puts a mother at risk of pregnancy outcomes. A previous study confirmed that even modest increases in maternal BMI were associated with increased risk of fetal death, stillbirth, and neonatal, perinatal and infant mortality [15]. Another study demonstrated that the strong seasonal pattern of conception in Bangladesh due to seasonal variations in coital frequency then to seasonality in nutritional status [16]. In addition to descriptive statistics of timing of first conception, life table has been constructed for having the probability of first conception waits in different duration of exposure months along with other summary measures. This study employed life table model because the dependent variable is duration type and those already been experienced first conception, known as uncensored, and also those who have not experienced first conception at the time of the survey, treated them as the censored cases.

In this study, life table for first conception interval is constructed to examine the distribution of first conception period in different exposure intervals taking into cognizance the censored cases, which may help to explain reproductive characteristics of all respondents. The probabilities by exposure intervals and other 
estimates are presented in the Table 2 and in the Table 3 accordingly. The measures used to estimate quantum of first conception in the function ${ }_{n} W_{x}$, the proportion of women having first conception by interval of $\mathrm{x}$ months from their date of first cohabitation. In the Table 2 , the value $\mathrm{W}_{18}=0.5377$ stats that about $54 \%$ women had first conception within 18 months of their first cohabitation. The results further indicate that $76 \%\left(\mathrm{~W}_{36}=0.7574\right), 87 \%\left(\mathrm{~W}_{60}=0.8704\right)$ and $90 \%\left(\mathrm{~W}_{72}=0.8970\right)$ ever married women had their first conception within 3, 5 and 6 years from the date of first cohabitation respectively. Here $\mathrm{W}_{72}$ can be treated as the most convenient indicator of the quantum of first conception interval because there are a small proportion of women likely to be conceived first time after a time span of 6 years from their date of first cohabitation

Applying linear interpolation technique, the quartiles are also computed to understand the scenario of timing of first conception in different quarters For example, first quartile $\left(\mathrm{Q}_{1}\right)$ can be obtained by linear interpolation between duration 0 and 6 months. Second quartile $\mathrm{Q}_{2}$ (Median) can be calculated from the duration 12 to 18 months and $\mathrm{Q}_{3}$ can be calculated from the duration of 24-30 months. From the estimated standardized quartiles, many of the statistical parameters in addition to those mentioned above can also be derived for discussion and interpretation of the results. For example, the tri-mean is a good measure of tempo as it is indicates average first conception interval of all respondents. The tri-mean can be calculated therefore as, $\mathrm{T}=\left(\mathrm{Q}_{1}+2 \mathrm{Q}_{2}+\mathrm{Q}_{3}\right) / 4$. Another measure of variability is the spread, which can be estimated as $\mathrm{S}=\mathrm{Q}_{3}-\mathrm{Q}_{1}$. 
Table 2. Abridge life table of timing of first conception among ever married women in Bangladesh

\begin{tabular}{|c|c|c|c|c|c|c|c|}
\hline 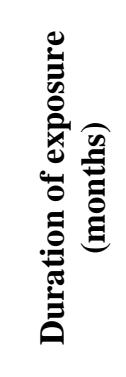 & 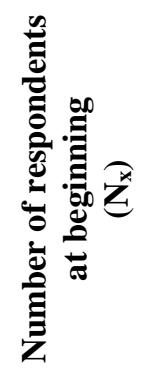 & 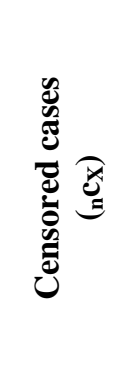 & 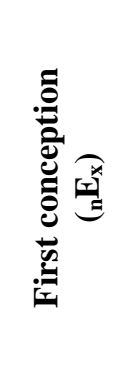 & 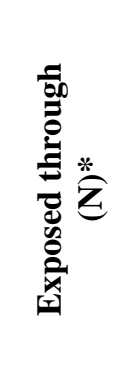 & 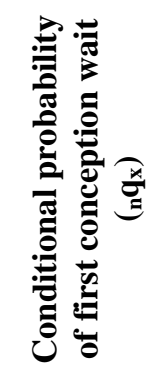 & 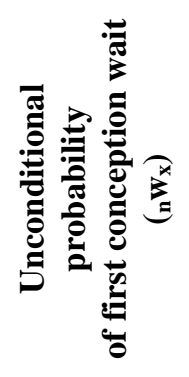 & 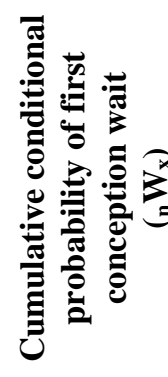 \\
\hline $0-6$ & 5491 & 287 & 1363 & 5204 & 0.2619 & 0.2619 & 0.2619 \\
\hline $6-12$ & 3841 & 175 & 715 & 3666 & 0.1950 & 0.1439 & 0.4058 \\
\hline $12-18$ & 2951 & 133 & 626 & 2818 & 0.2221 & 0.1319 & 0.5377 \\
\hline $18-24$ & 2192 & 90 & 418 & 2102 & 0.1989 & 0.0919 & 0.6296 \\
\hline $24-30$ & 1684 & 97 & 294 & 1587 & 0.1853 & 0.0686 & 0.6982 \\
\hline $30-36$ & 1293 & 60 & 242 & 1233 & 0.1963 & 0.0592 & 0.7574 \\
\hline $36-42$ & 991 & 48 & 165 & 943 & 0.1749 & 0.0424 & 0.7998 \\
\hline $42-48$ & 778 & 49 & 111 & 729 & 0.1523 & 0.0305 & 0.8303 \\
\hline $48-54$ & 618 & 31 & 60 & 587 & 0.1022 & 0.0173 & 0.8476 \\
\hline $54-60$ & 527 & 38 & 73 & 489 & 0.1493 & 0.0228 & 0.8704 \\
\hline $60-66$ & 416 & 24 & 42 & 392 & 0.1071 & 0.0139 & 0.8843 \\
\hline $66-72$ & 350 & 21 & 36 & 329 & 0.1094 & 0.0127 & 0.8970 \\
\hline $72-78$ & 293 & 15 & 26 & 278 & 0.0935 & 0.0096 & 0.9066 \\
\hline $78-84$ & 252 & 9 & 21 & 243 & 0.0864 & 0.0080 & 0.9146 \\
\hline $84-90$ & 222 & 16 & 14 & 206 & 0.0679 & 0.0058 & 0.9204 \\
\hline $90+$ & 192 & 118 & 74 & 74 & 1 & 0.0796 & 1 \\
\hline \multicolumn{2}{|c|}{ Total Cases } & 1211 & 4280 & & & & \\
\hline
\end{tabular}


Findings from the Table 3 reflect that tri-mean of first conception interval is estimated at about 16 months, median is about 14 months and the spread of first conception interval is 24 months. Based on the tri-mean and median, the distribution of first conception waits among Bangladeshi women is positively skewed as per Bowley's coefficient of skewness which is in agreement with Pearson's coefficient. The higher value of spread (23.78) indicates that the pattern of first conception waits is extremely heterogeneous, which also in the line of overall standard deviation.

Table 3. Descriptive measures of first conception interval of ever married respondents by life table technique

\begin{tabular}{cc}
\hline Summary Measures & First Conception \\
\hline $\mathrm{W}_{6}$ & 0.2619 \\
$\mathrm{~W}_{12}$ & 0.4058 \\
$\mathrm{~W}_{18}$ & 0.5377 \\
$\mathrm{~W}_{24}$ & 0.6296 \\
$\mathrm{~W}_{36}$ & 0.7574 \\
$\mathrm{~W}_{72}$ ( quantum) & 0.8970 \\
$\mathrm{Q}_{1}$ & 4.995 (months) \\
$\mathrm{Q}_{2}=$ median & $13.62(14$ months $)$ \\
$\mathrm{Q}_{3}$ & $28.77(29$ months $)$ \\
Tri-mean(T) & $15.5(16$ months) \\
Spread(S) & $23.78(24$ months \\
No. of cases & $5491\left(4281+1211^{*}\right)$ \\
\hline
\end{tabular}

* Censored cases

In addition to the findings presented in Table-3, the cumulative probability of first conception by exposure intervals are also depicted in the Figure-1 for easy understanding of probability of having first conception in the different points of exposure duration of first conception. Figure-1 at a glance reveals that cumulative probability of having first conception gradually increases up to the 60 months of marital duration. The findings of first conception interval obtained from the life table 
analysis show that distribution of timing of first conception is heterogeneous because $\mathrm{Q}_{1}$ and $\mathrm{Q}_{3}$ are not equidistant from the median. Results of tri-mean and $\mathrm{Q}_{2}$ indicate that $\left(\mathrm{T}>\mathrm{Q}_{2}\right)$ the shape of the distribution is positively skewed. Summary measures further illustrate that mean first conception interval among Bangladeshi women is longer.

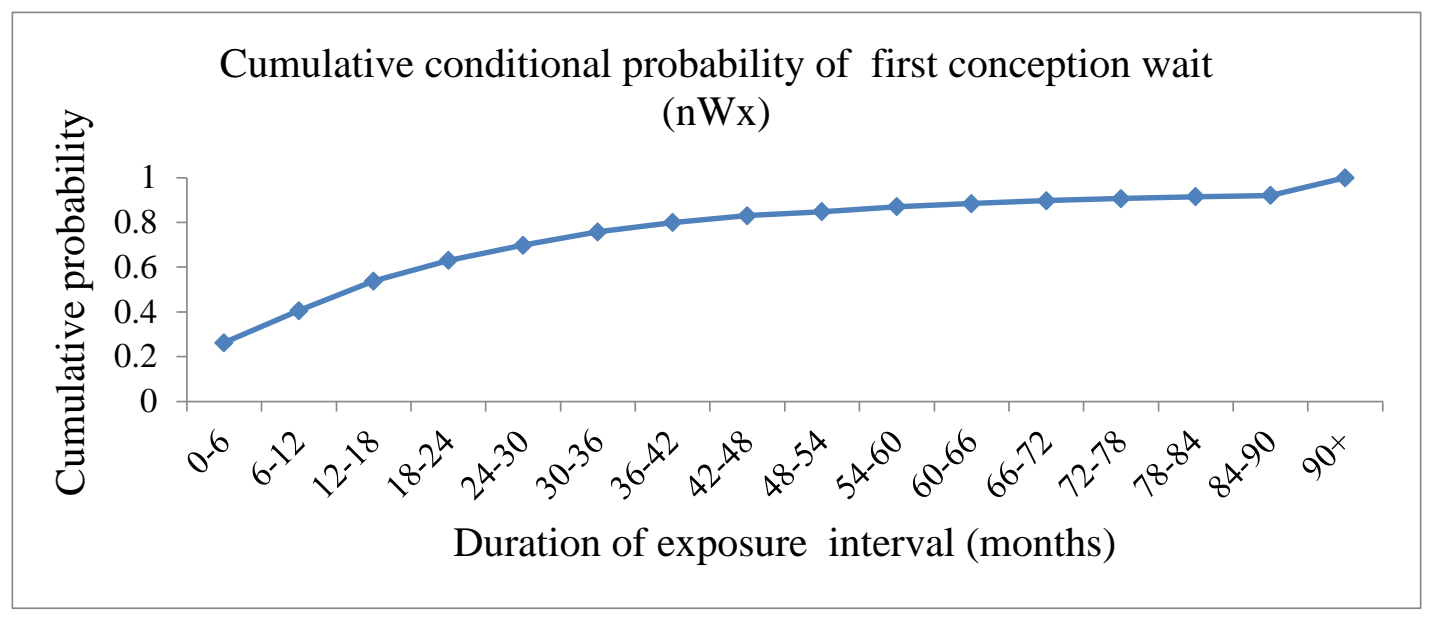

Figure 1: Life table function of timing of first conception

The cumulative probability of having first conception shows that about $76 \%$ ever married women become conceived within 3 years of their first cohabitation, although the mean age at first cohabitation of the Bangladeshi females is only 15.86 years [16]. A substantial difference has been observed between mean first conception interval and tri-mean. The reason behind the difference is that a considerable number of women who have no conception at the date of the interview and who may have cohabitated neighborhood of survey date. Moreover, existence of outliers in the conception intervals among the respondents belong to last quarter may be the possible explanation for the longer mean conception wait compared to tri-mean. 


\section{Conclusion}

Analysis of waiting time for first conception shows females reproductive characteristics and fertility at the early stage of marriage. In this study the overall first mean conception wait among ever married women is found to 19.13 months. It is found prolonged among the respondents living in Dhaka, among illiterates, whose age at first cohabitation is up to 9 years; belong to overweight stratum and among the poor. The shorter conception waits are estimated among those who are highly educated, belong to high socioeconomic status, have normal weight, residing in Chittagong division and among recent marriage cohort. The shape characteristics of the distribution of first conception delay is positively skewed and leptokurtic. Value of spread (23.78) reveals that there exists extreme heterogeneity in first conception intervals. Distribution of conception waits show that the tri-mean is 4 months less than overall mean. One of the possible explanations of shorter tri-mean is that the date of first cohabitation of some respondents may be the neighborhood of date of survey while longer overall fist conception interval may be attributed with the existence of outliers in the conception waits. Moreover, misreporting of exact date of vital events due to lack of complete vital registration may distort its computation. Unreported early pregnancy loss may be the possible reason for longer first conception interval. Although the first conception wait is estimated as relatively longer but findings in Table 3 reveal that about $41 \%$ respondents had their first conception within one year $\left(\mathrm{W}_{12}=0.4058\right)$ of their marital duration and it is about $76 \%$ within 3 years $\left(\mathrm{W}_{36}=0.7574\right)$ from their date of first cohabitation. Based on the findings it can be concluded that longer mean conception interval after first cohabitation may not be an only reason for longer subsequent conception intervals and reduction of fertility. Likewise, shorter first conception interval may not be an only cause for the same. For instance, summary measures of conception wait and values of log-rank test statistic in Table 1 show that mean first conception interval is found significantly longer among those who are illiterate, whose age at first cohabitation is lower and respondents belong to poor socio economic stratum. On the contrary, fertility of aforesaid groups is found higher in Bangladesh [13]. In the 
analytical aspect, it may be pointed out that the estimates obtained in this study may be slightly inflated or deflated due to misreporting of relevant vital events. These may happen at the time of retrospective survey due to memory lapse, lack of complete vital registration and longitudinal inquiry. Therefore, implementation of complete vital registration system across the country is immensely important to overcome the aforementioned issues. Thus, based on the findings of this study it can be suggested that the existing Marriage Act should be implemented properly for rising the age at first cohabitation. Nevertheless, fruitful policies should be made by the policy makers and planners to increase the literacy rate, improving the socioeconomic status and to ensure the balanced nutritional status for normal first conception delay and achieving the ultimate goal for replacement level fertility in Bangladesh.

\section{References}

[1] R. G. Potter and M.P. Parker: Population Studies, 1964, 18, 99.

[2] D. C. Nath, K. C. Land and K. K. Singh: Genus, 1995, 1-2, 95.

[3] W. H. James: Social Biology, 1979, 26(4), 330.

[4] S. S. Sharma, A. K. Shukla and A. K. Tiwari: Research Communication, 2007, 1(2-3), 26.

[5] D. J. Teachman and D. A. Heckert: Demography, 1985, 22(2), 185.

[6] J. Hajnal: Population and Development Review, 1982, 8(3), 449.

[7] R. Lesthaeghe: Population Studies, 1971, 25(3), 415.

[8] J. Bongaarts and R. G. Potter, Fertility, Biology and Behavior: An Analysis of the Proximate Determinants of Fertility. Academic Press, New York, 1983.

[9] A. K. Jain: Population Studies, 1969, 23, 69.

[10] N. Zare, B. Nouri and M. Pavareh: International Journal of Reproductive BioMedicine, 2017, 15(10),11.

[11] N. S. Singh, N. Sanajaoba and R. K. Narendra : Stud Home Commun Sci, 2011, 5, 7.

[12] M. Vanlaldingham: Social Biology, 1993, 40(3-4), 213.

[13] Mitra and Associates and Macro International, Bangladesh Demographic and Health Survey, Dhaka, Bangladesh and Calverton, Maryland, USA, 2014. 
92 Md Abdul Karim and Afia Azimoon

[14] United Nations: "Patterns of First Marriage, Timing and Prevalence", 1990.

[15] D. Aune, O. D. Saugstad, T, Henriksen and S. Tonstad: "Americam Medical Association", JAMA, 2014, 311(15), 1536.

[16] J. A. Menken: Demography, 1979, 16(1), 103.

The Chittagong Univ. J. Sc. Vol. 43(1), 2021 